\title{
CALIDAD DE VIDA Y ADHERENCIA AL TRATAMIENTO EN PACIENTES CON ENFERMEDAD DE PARKINSON
}

\section{QUALITY OF LIFE AND TREATMENT ADHERENCE IN PARKINSON'S DISEASE PATIENTS}

\section{TITULO CORTO: CALIDAD DE VIDA Y ADHERENCIA AL TRATAMIENTO EN PACIENTES}

\section{Sara Mínguez-Mínguez ${ }^{1}$, Susana García-Muñozguren², Julián Solís-García del Pozo ${ }^{3}$ y Joaquín Jordán ${ }^{4}$}

Recibido en febrero 20 de 2015

Aceptado en mayo 26 de 2015

\section{RESUMEN}

La calidad de vida y la adherencia al tratamiento poseen una gran relevancia en aquellos pacientes que presentan enfermedades crónicas. El objetivo de este trabajo es conocer y evaluar ambos parámetros, en una población de pacientes de enfermedad de Parkinson. Para ello, se ha realizado un estudio transversal, a través de los cuestionarios de PDQ-39 y de Morisky-Green, en 95 pacientes de la citada enfermedad pertenecientes a alguna de las asociaciones de pacientes y familias de la provincia de Albacete. Los datos muestran una puntuación media de 33,47\% en el PDQ-39, siendo los dominios de peor puntuación los referidos a movilidad y malestar físico. Por otro lado, un 31,6\% de los pacientes se clasificaron como adherentes al tratamiento. Respecto a este parámetro, constatamos que, el factor principal relacionado con una adecuada adherencia, fue la importancia dada a su medicación por el propio paciente. No se ha encontrado ninguna asociación entre adherencia y calidad de vida.

Palabras clave: Enfermedad de Parkinson; Calidad de Vida; Cumplimiento de la Medicación. (Fuente DeCS)

\section{AвSTRACT}

Quality of life and adherence to treatment are parameters of high relevance in those patients with chronic diseases. The aim of this study was to ascertain the quality of life and adherence to treatment of Parkinson's disease patients. To this end, we performed a cross sectional study in patients diagnosed with Parkinson's disease who belong to

1. Farmacéutica. Doctora en el departamento de Ciencias Médicas. Facultad de Medicina de Albacete. Universidad de Castilla-La Mancha (UCLM). España. Correo electrónico: minguezsara@gmail.com

2. Médico especialista en neurología. Servicio Neurología. Complejo Hospitalario Universitario de Albacete. Albacete. España. Correo electrónico: Sugarmu@gmail.com

3. Médico especialista en Medicina Interna. Doctor en Medicina. Jefe de Servicio de Medicina Interna. Hospital General de Villarrobledo (Albacete). España. Correo electrónico: julianeloysolis@gmail.com

4. Farmacéutico. Doctor en Farmacia. Profesor titular de farmacología del departamento de Ciencias Médicas. Facultad de Medicina de Albacete. Universidad de Castilla-La Mancha (UCLM). Grupo de Neurofarmacología. Instituto de Investigación en Discapacidades Neurológicas-UCLM. España. Correo electrónico: Joaquin.Jordan@uclm.es 
one of Albacete's associations of patients and their families. The PDQ-39 and Morisky-Green questioners were used to determine the quality of life and adherence to treatment for a sample of 95 patients. Our data showed an average score of $33.47 \%$ in the PDQ-39, being the worst score domains mobility and bodily discomfort. On the other hand, a $31.6 \%$ of the patients were classified as adherent to treatment. The main factor associated with adequate adherence is the importance given to the medication by the patient. We found no association between adherence and quality of life.

Keywords: Parkinson disease; Quality of life; Medication adherence. (Fuente DeCS)

\section{INTRODUCCIÓN}

A sociado al hecho de que una enfermedad puede provocar discapacidad, la Organización Mundial de la Salud (OMS) define calidad de vida como la "la percepción del individuo de su posición en la vida en el contexto de la cultura y sistema de valores en los que vive y en relación con sus objetivos, expectativas, estándares y preocupaciones" ${ }^{1}$. La cuantificación de la calidad de vida de un paciente, relacionada con su salud, puede resultar de gran ayuda para analizar aspectos no detectables de otra manera como el estado psicológico, o el efecto de la enfermedad sobre las relaciones sociales o sobre las creencias del enfermo, o la discapacidad producida o el estado físico. Para su cuantificación se han desarrollado diferentes instrumentos (genéricos y específicos), entre los que destaca el Sickness Impact Profile, el Nottingham Health Profile y el Short Form Health Survey ${ }^{2-4}$. Sin embargo, cuando se ha intentado aplicar alguno de estos instrumentos a una patología en concreto, se ha observado que ofrecen un potencial escaso, presentando como problema principal poder medir áreas específicas y por tanto su insuficiente sensibilidad al cambio.

Este parece ser el caso de la enfermedad de Parkinson (EP). Esta es la segunda enfermedad neurodegenerativa más frecuente y está caracterizada por síntomas motores como la rigidez, el temblor o la acinesia ${ }^{5}$. Se trata de una enfermedad crónica, progresiva e incapacitante, donde la calidad de vida de los pacientes se ve comprometida a lo largo de su evolución. Para analizar la calidad de vida de estos pacientes en el año 1995, en la Universidad de Oxford, se creó el cuestionario PDQ-39 (del inglés Parkinson Disease Questionnary-39) ${ }^{6}$. Éste evalúa 39 parámetros divididos en ocho grupos de cuestiones, y abarca áreas como: movilidad, actividades domésticas, bienestar emocional, estigma social, apoyo social, cognición, comunicación y malestar corporal ${ }^{7,8}$. Hoy día disponemos una versión del PDQ-39 validada en español ${ }^{9}$.

Otro de los parámetros asociados a la conducta de un paciente es el expresado como cumplimiento terapéutico, observancia terapéutica o adherencia al tratamiento. Así, se puede definir la adherencia al tratamiento como el grado hasta el cual la conducta del paciente, en términos de tomar medicamentos, seguir las dietas, o los cambios en el estilo de vida, coinciden con las prescripciones clínicas $^{10,11}$. Su determinación resulta muy importante para comprender la respuesta o no a un tratamiento pautado ${ }^{12-14}$.

De una forma más concreta, entre los diferentes métodos utilizados para su determinación en pacientes con EP, destacan: el recuento de pastillas, la monitorización electrónica, la medición de metabolitos en diferentes líquidos orgánicos, y el cuestionario de Morisky-Green. El cuestionario de Morisky-Green es un instrumento diseñado para valorar si el paciente adopta actitudes correctas en relación con el tratamiento prescrito ${ }^{12,15}$.

En un estudio previo, Grosset et al. ${ }^{13}$, describieron una posible asociación entre variables de calidad de vida y adherencia al tratamiento. Por todo ello, el presente estudio pretende determinar la posible asociación entre la percepción de calidad de vida relacionada con la salud y adherencia al tratamiento en pacientes con enfermedad de Parkinson. Así mismo, se profundiza en la existencia de relaciones entre calidad de vida, adherencia al tratamiento y las características poblacionales de los pacientes de EP.

\section{MATERIAL Y MÉTODOS}

Se ha realizado un estudio observacional y transversal en los pacientes diagnosticados con la enfermedad de 
Parkinson que pertenecían a alguna de las siguientes asociaciones de pacientes y familias de la provincia de Albacete, (España): Albacete, Almansa, Hellín, La Roda y Villarrobledo. Los criterios de inclusión fueron: edad superior a 18 años de edad con diagnóstico clínico de enfermedad de Parkinson basado en la presencia de los síntomas cardinales de temblor, acinesia y rigidez, y estar en tratamiento con algún fármaco antiparkinsoniano. Los criterios de exclusión fueron: la existencia de discapacidad debida a otras enfermedades neurológicas (como ictus o enfermedad de Alzheimer), enfermedad concomitante grave (como: neoplasia, broncopatía o cardiopatía grave), o el rechazo a participar en el estudio. Toda la información, autocomunicada, se recogió mediante entrevista personal llevada a cabo por un único investigador (SMM) previamente entrenado.

Se recogieron variables sociodemográficas (edad, sexo, estado civil), de nuestra cohorte y se realizó un cuestionario (Tabla 1) que incluía preguntas sobre actitudes ante la medicación antiparkinsoniana prescrita en dichos pacientes.

Tabla 1: Actitudes ante la medicación. Cuestionario

1- ¿Piensa que su tratamiento para el Parkinson es fácil de tomar?

$\square$ Muy fácil $\quad \square$ Más o menos fácil $\quad \square$ Nada fácil

2- ¿Ha recibido indicaciones precisas de cómo tomar su medicación para el Parkinson?

$\square$ Sí $\quad \square$ No $\quad \square$ No lo sé

3- ¿Son las instrucciones de cómo tomar su medicación para el Parkinson un inconveniente para usted?

$\square$ Muy inconveniente $\quad \square$ Más o menos inconveniente $\quad \square$ No son un inconveniente

4- ¿Alguna vez se olvida de tomar su medicación para el Parkinson?

$\square$ Nunca $\quad \square$ A veces $\quad \square$ A menudo

5- ¿Cómo se encuentra de motivado para tomar su medicación para el Parkinson?

$\square$ Muy motivado.

口Un poco motivado.

$\square$ Nada motivado

6- ¿Qué le motiva a tomar su medicación para el Parkinson? (puede marcar más de una casilla)

$\square$ Mi médico.

口La gente de mi entorno.

口Tengo miedo a empeorar.

$\square$ Para mantener la salud mental.

$\square$ Es fácil de tomar.

$\square$ No hay nada en particular.

¿Otro (especificar):

7- "Mi medicación para el Parkinson es importante para mi salud"

$\square$ Sí, completamente de acuerdo.

$\square$ Más o menos de acuerdo.

$\square$ No, en absoluto.

- Calidad de vida: Se evaluó mediante el cuestionario de calidad de vida específico para enfermedad de Parkinson PDQ-396,9. Tiene 39 ítems agrupados en 8 dominios que son: movilidad, actividades de la vida diaria, bienestar emocional, si su enfermedad supone estigma, apoyo social, deterioro cognitivo, dificultad para comunicación y malestar físico. La puntuación del PDQ-39 se ofrece como un perfil en donde para cada dimensión el valor final del resultado es la suma de cada valor dividido por el número total de preguntas por dimensión. El resultado obtenido se multiplicará por 100 , así cada dimensión del cuestionario puntuará de 0 a 100, en donde 0 es la mejor y 100 la peor puntuación.

- Adherencia al tratamiento: Se evaluó gracias al cuestionario de Morisky-Green ${ }^{15}$. Éste consta de 4 preguntas, por cada respuesta adecuada a cada una 
de estas preguntas el cuestionario suma 1 punto. Se considera que la adherencia al tratamiento es alta si la puntuación del cuestionario es 4 . Una puntuación de 2-3 indica una adherencia moderada al tratamiento. Una puntuación por debajo de 2 indica una adherencia baja. Los pacientes con puntuación por debajo de 4 fueron considerados no adherentes, en consonancia con lo publicado en otros estudios.

Las respuestas de los participantes fueron introducidas en una base de datos anónima, para proceder posteriormente a su depuración y análisis. Inicialmente se realizó un estudio descriptivo de la población y del resultado de los cuestionarios, así como de los fármacos que usan estos pacientes. Las variables categóricas se describen como n (\%). Las variables cuantitativas se describen como media \pm desviación standard y/o como mediana (rango), según la distribución normal o no de la variable. La normalidad de la variable se comprobó con el test de Kolmogorov-Smirnov. Posteriormente y para comprobar la existencia de asociaciones entre la adherencia al tratamiento y diferentes variables sociodemográficas, se realizaron comparaciones utilizando el test de MannWhitney para la comparación de dos grupos con una variable cuantitativa. Se ha usado el test de la $\chi^{2}$ cuando las variables eran cualitativas y el test de Fisher cuando ha sido preciso. Por último, se han empleado estos mismos test estadísticos para la comparación de la adherencia con las diferentes dimensiones de la calidad de vida usadas en el cuestionario PDQ-39. Se tomó como nivel de significación $\mathrm{p}<0,05$. Se utilizó para realizar los cálculos el programa SPSS.

Todas las personas que colaboraron en este estudio lo hicieron de manera voluntaria. No se recogieron ni almacenaron datos personales de identificación de los pacientes. Los datos recogidos no se usaron con otra finalidad que no fuera la realización de este estudio.

\section{RESULTADOS}

\section{Variables sociodemográficas}

Se entrevistaron 101 pacientes, de los cuales 6 no fueron capaces de completar los cuestionarios por lo que fueron excluidos del estudio. Por tanto, un total de 95 pacientes se incluyeron finalmente en nuestra cohorte. El 58,9\% de los pacientes fueron varones, con una edad media de 71,7 \pm 8,75 años. El 75,8\% estaba casado, un 93,7\% jubilados, y vivían en su propia casa el 91,6\%. La medicación era administrada por el propio paciente en el 58,9\% de los casos.

La mayoría $(73,7 \%)$ de los pacientes consideran que su medicación es muy fácil de tomar, y el $94,7 \%$ han recibido por parte del personal sanitario, las indicaciones precisas para su correcto uso. Un 48,4\% declaraban que no se olvidaban nunca de tomar su medicación, mientras que se olvidaba a menudo el 2,1\%. Un 69,5\% de los pacientes declararon sentirse muy motivados para tomar su medicación. El 50,5\% contestaron que la mayor motivación para tomar su medicación es el miedo a empeorar. Un 92,6\% está convencido de que su medicación para el Parkinson es importante para su salud.

\section{Calidad de vida}

El resultado de la puntuación de cada uno de los ocho dominios del cuestionario PDQ-39 se representa en el gráfico de cajas de la figura 1. La puntuación media del PDQ-39 de la población estudiada fue de 33,47\% $\pm 19,05$. A continuación, con el fin de reconocer las posibles asociaciones entre los dominios del PDQ-39 y las características sociodemográficas de los pacientes, se realizó un análisis bivariante.

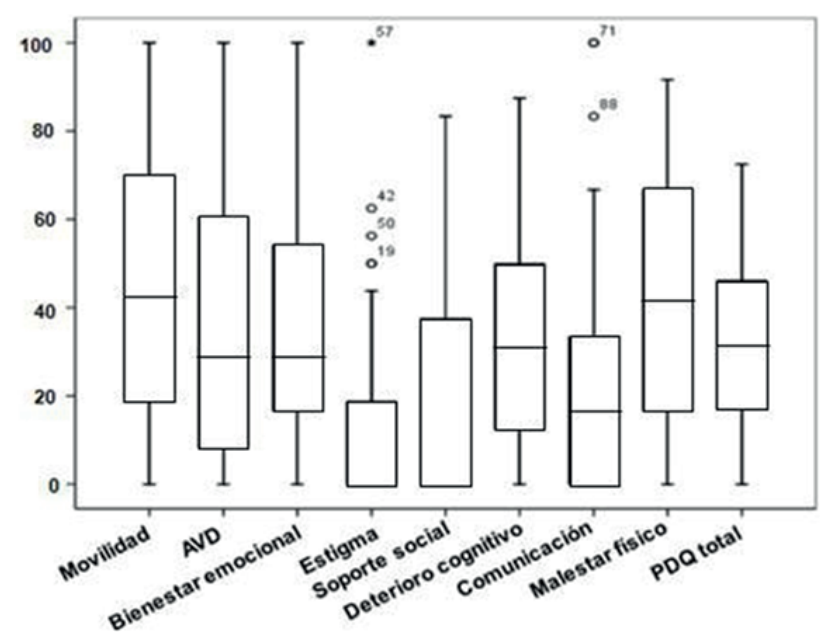

Figura 1. Calidad de vida en pacientes con enfermedad de Parkinson. Puntuaciones del cuestionario PDQ-39

Así, se encontró como: a.) las mujeres suelen tener peor puntuación que los hombres en los dominios del PDQ39, movilidad ( $p=0,001)$, actividades de la vida diaria 
$(p=0,051)$, bienestar emocional $(p=0,009)$, soporte social $(\mathrm{p}<0,001)$ y discomfort o malestar físico $(\mathrm{p}=0,001)$; b. $)$ aquellos pacientes a los que otra persona les administra la medicación presentan peor puntuación en los apartados de movilidad $(\mathrm{p}<0,001)$, actividades de la vida diaria ( $p<0,001)$ y peor capacidad de comunicación $(\mathrm{p}=0,056)$.

\section{Adherencia al tratamiento}

La adherencia al tratamiento se midió gracias al cuestionario de Morisky-Green. Se consideró como no adherentes aquellos pacientes con una puntuación menor de 4 . Según este criterio, 30 pacientes $(31,6 \%)$ eran adherentes al tratamiento.

El análisis bivariante mostró cómo los pacientes que "más convencidos se mostraron de la importancia de su medicación antiparkinsoniana para su salud", fueron adherentes con más frecuencia $(p=0,031)$. No se ha encontrado asociación entre la adherencia al tratamiento y el sexo $(p=0,228)$, la edad $(p=0,93)$, el estado civil $(p=0,531)$ o dependencia a quien era la persona responsable de la administración de la medicación $(\mathrm{p}=0,098)$.

\section{Asociación entre calidad de vida y adherencia al tratamiento}

Por último, se intentó establecer si existe algún tipo de asociación entre los resultados de calidad de vida y adherencia al tratamiento. De esta manera, se encontró la existencia de una única asociación, la que surge con el dominio centrado en el deterioro cognitivo. Asociándose un menor deterioro cognitivo con una mayor adherencia al tratamiento $(p=0,021)$. No se ha encontrado otra relación entre los diferentes dominios del PDQ-39 y la adherencia al tratamiento.

\section{DISCUSIÓN}

La enfermedad de Parkinson se caracteriza por ser de evolución progresiva e incapacitante, pudiendo alterar la calidad de vida de sus pacientes. En el presente trabajo se han utilizado los cuestionaros de PQD-39 y de MorinskyGreen para conocer parámetros de calidad de vida y de adherencia al tratamiento y sus posibles asociaciones en una población de pacientes de enfermedad de Parkinson que pertenecen a asociaciones de familias y pacientes de la provincia de Albacete.
Se ha observado que la mayoría de los pacientes entrevistados declaraban hacer recibido suficiente información acerca de la forma en la que debe tomarse la medicación y se encontraban muy motivados para hacerlo; siendo además el propio enfermo el encargado en la mayoría de los casos de su propia medicación. El miedo a empeorar es una de las mayores motivaciones de los pacientes para tomar correctamente su medicación, que perciben mayoritariamente como importante para su salud.

En este trabajo se ha utilizado como instrumento de medida de la calidad de vida el PDQ-39. Esta decisión fue tomada en base a una revisión sistemática de los instrumentos de medición de calidad de vida en la enfermedad de Parkinson, que sugiere como el más adecuado al PDQ39, debido a su validez de contenido y de constructo, seguido del PDQL ${ }^{16}$. Las peores puntuaciones obtenidas fueron en los dominios referentes a la movilidad, las actividades de la vida diaria y el bienestar emocional. En línea con esta observación, Rodríguez Violante encontró que estos mismos dominios son los que presentan peores puntuaciones en su estudio en México en pacientes con enfermedad de Parkinson ${ }^{17}$. Así mismo se ha encontrado asociación entre la peor puntuación en movilidad, actividades de la vida diaria y comunicación y el hecho de que la medicación se la administre otra persona.

Resulta llamativa la asociación entre peores puntuaciones en el PDQ-39 y el sexo femenino. Este hecho ha sido descrito también en el estudio de Carod-Artal ${ }^{18}$. En dicho estudio, las mujeres eran de mayor edad que los hombres y estaban en estadios más avanzados de la enfermedad. En el presente estudio, el tiempo de evolución de la enfermedad era mayor en las mujeres, lo que también podría relacionarse con enfermedad más avanzada, explicándose así esta asociación. Sin embargo, no se puede dejar a un lado que otros estudios como el de Navarro-Peternella no encuentra claras diferencias de puntuación en el PDQ-39 respecto al sexo ${ }^{19}$.

Uno de las conclusiones más relevantes de este estudio es el bajo porcentaje de pacientes adherentes al tratamiento $(31.6 \%)$. Llama la atención que este bajo porcentaje es similar al encontrado en otros estudios que utilizan este mismo cuestionario en pacientes de población anciana $^{20,21}$. No obstante, en pacientes de EP, con el mismo cuestionario, se obtuvo un porcentaje de pacientes adherentes más alto $(60.4 \%)^{22}$. 
El presente estudio puede presentar algunas limitaciones. Nuestra cohorte es pequeña y restringida a voluntarios pertenecientes a asociaciones, por lo que pacientes con enfermedad más evolucionada y grave pueden no estar representados en nuestro estudio. La posible relación entre el estadio de la enfermedad, los síntomas que el paciente presenta y el tratamiento farmacológico con los cuestionarios ha quedado fuera de nuestro objetivo general de estudio. Si bien, otros estudios han puesto de manifiesto que tanto los síntomas motores como los no motores repercuten en la calidad de vida del paciente y que incluso la mitad de los pacientes refiere que esta disfunción no motora afecta de forma "importante" y "muy importante" sus actividades de vida diaria ${ }^{23}$.

A pesar de estas limitaciones, este trabajo nos permite aproximarnos a la realidad del impacto de la enfermedad de Parkinson en la vida de los que la sufren y a la vez de cómo los propios afectados ven su enfermedad y su tratamiento.

\section{REFERENCIAS BIBLIOGRÁFICAS}

1. The World Health Organization Quality of Life assessment (WHOQOL): position paper from the World Health Organization. SocSci Med. 1995; 41(10): 1403-9.

2. Bergner M, Bobbitt RA, Kressel S, Pollard WE, Gilson BS, Morris JR. The sickness impact profile: conceptual formulation and methodology for the development of a health status measure. Int J Health Serv. 1976; 6 (3): 393-415.

3. Karlsen KH, Larsen JP, Tandberg E, Maland JG. Quality of life measurements in patients with Parkinson's disease: A community-based study. Eur J Neurol. 1998; 5 (5): 443-50.

4. Ware JE, Sherbourne CD. The MOS 36-item short-form health survey (SF-36). I. Conceptual framework and itemselection. Med Care. 1992; 30 (6): 473-83.

5. Martínez-Lapiscina E, Pulido-Fontes L, Erro-Aguirre ME. Manejo terapéutico de las fluctuaciones motoras en la enfermedad de Parkinson. Rev Neurol. 2012; 54 (Suppl 5): S25-S32.

6. Peto V, Jenkinson C, Fitzpatrick R. PDQ-39: a review of the development, validation and application of a Parkinson's disease quality of life questionnaire and its associated measures. J Neurol. 1998; 245 (Suppl 1): S10-4.
7. Bushnell DM, Martin ML. Quality of life and Parkinson's disease: translation and validation of the US Parkinson's Disease Questionnaire (PDQ-39). Qual Life Res.1999; 8, 345-50.

8. Jenkinson C, Fitzpatrick R, Peto V, Greenhall R, Hyman N. The Parkinson's Disease Questionnaire (PDQ-39): development and validation of a Parkinson's disease summary index score. Age Ageing. 1997; 26, 353-7.

9. Martinez-Martin P, FradesPayo B. Quality of life in Parkinson's disease: validation study of the PDQ39 Spanish version. The Grupo Centro for Study of Movement Disorders. J Neurol. 1998; 245 (Suppl 1): S34-8.

10. Rigueira-García AI. Cumplimiento terapéutico ¿qué conocemos en España? Atención Primaria, 2001; 27 (8): 559-68.

11. Sackett DL, Haynes RB, Gibson ES, Hackett BC, Taylor DW, Roberts RS, et al. Randomised clinical trial of strategies for improving medication compliance in primary hypertension. Lancet. 1975; 1 (7918): 1205-7.

12. Elm JJ, Kamp C, Tilley BC, Guimaraes P, Fraser D, Deppen P, et al. Self-reported adherence versus pill count in Parkinson's disease: the NET-PD experience. Mov Disord. 2007; 22 (6): 822-7.

13. Grosset D. Therapy adherence issues in Parkinson's disease. J Neurol Sci. 2010; 289 (1-2): 115-8.

14. Grosset KA, Bone I, Reid JL, Grosset D. Measuring therapy adherence in Parkinson's disease: a comparison of methods. J Neurol Neurosurg Psychiatry. 2006; 77 (2): 249-51.

15. Morisky DE, Green LW, Levine DM. Concurrent and predictive validity of a self-reported measure of medication adherence. Med Care. 1986; 24 (1): 67-74.

16. Forsaa EB, Larsen JP, Wentzel-Larsen T, Herlofson K, Alves G. Predictors and course of health-related quality of life in Parkinson's disease. Mov Disord. 2008; 23 (10): 1420-7.

17. Rodriguez-Violante M, Cervantes-Arriaga A, Corona T, Martinez-Ramirez D, Morales-Briceno H, MartinezMartin P. Clinical determinants of health-related quality of life in Mexican patients with Parkinson's disease. Arch Med Res. 2013; 44 (2): 110-4.

18. Carod-Artal FJ, Vargas AP, Martinez-Martin P. Determinants of quality of life in Brazilian patients with Parkinson's disease. Mov Disord. 2007; 22 (10): 1408-15.

19. Navarro-Peternella FM, Marcon SS. Quality of life of a person with Parkinson's disease and the relationship between the time of evolution and the severity of the disease. Rev Lat Am Enfermagem.2012; 20 (2): 384-91. 
20. Lee VW, Pang KK, Hui KC, Kwok JC, Leung SL, Yu DS, et al. Medication adherence: Is it a hidden drug-related problem in hidden elderly? Geriatr Gerontol Int. 2013; 13(4):978-85.

21. Markotic F, Cerni Obrdalj E, Zalihic A, Pehar R, Hadziosmanovic Z, Pivic G, et al. Adherence to pharmacological treatment of chronic nonmalignant pain in individuals aged 65 and older. Pain Med. 2013; 14 (2): $247-56$.
22. Valldeoriola F, Coronell C, Pont C, Buongiorno MT, Camara A, Gaig C, et al. Socio-demographic and clinical factors influencing the adherence to treatment in Parkinson's disease: the ADHESON study. Eur J Neurol. 2011; 18 (7): 980-7.

23. Pfeiffer RF. Parkinson disease. Nonmotor symptoms in Parkinson disease: the PRIAMO study. Nat Rev Neurol. 2009; 5 (10): 531-2.

Para citar este artículo: Mínguez-Mínguez S, García-Muñozguren S, Solís-García del Pozo J, Jordán J. Calidad de vida y adherencia al tratamiento en pacientes con enfermedad de Parkinson. Duazary. 2015 dic; 12 (2): 133 - 139 